NO. 2000-3000
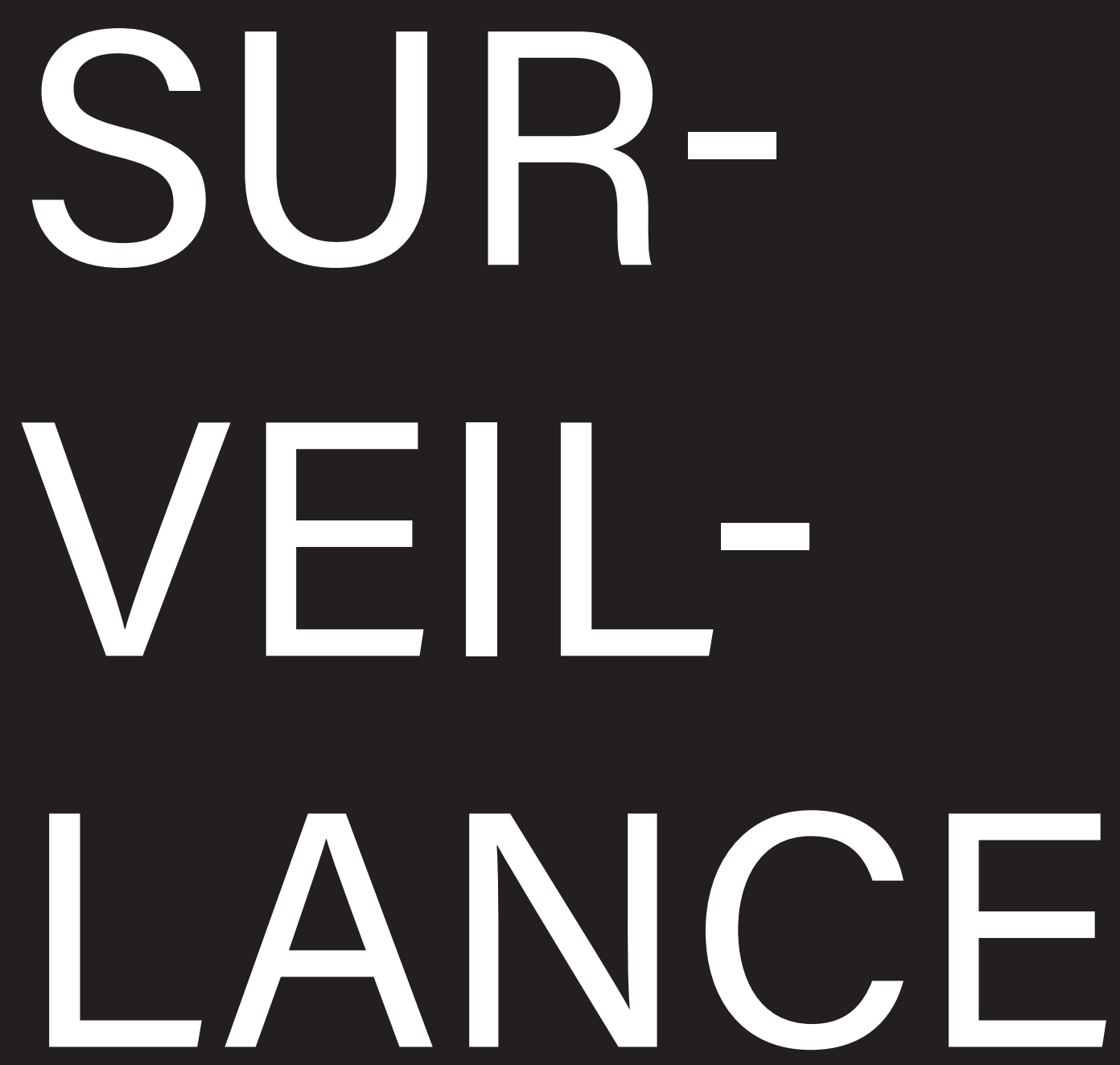

\title{
FROM THE THIRD MILLENNIUM
}

\section{YUNG AU}

yung.au@oii.ox.ac.uk

University of Oxford, UK 



\section{The Third Millennium}

Welcome to the non-definitive guide to the history of the third millennium.

This archival project aims to be a living document of fragments, narratives, and artefacts of the astronomical years of 2000-3000 (or 12000-13000 Holocene Era/Human Era). As another chapter of the long, deep, and convoluted histories of humanity, our understanding of life during this time is still very limited. It is for this reason that this archive is an open and collaborative one. Please feel free to edit and submit any knowledge fragments to expand our compendium on what life was like in one of the earliest millennia of human life on earth.

Much of the squabbles, fixations, and anxiety from this age may seem alien in today's society. This compendium hopes to shed some light on what this era looked like from the inside. It should be noted that there is an overwhelming amount of data, accounts, counter-accounts, counter-counter accounts, and counter-counter-counter accounts from this period that we are still parsing to this day. There is thus no one single account of this historical period but a multiplicity of stories.

Here are today's featured article fragments to get you started on your timehopping adventures:

01. "Selling Quickness"

02. "An Era of Perpetual Records"

03. "Optimise"

04. "Outlawed Taylorist Innovations" 


\section{SELLING}

\section{QUICKNESS}

Time moved from days, hours, seconds, miliseconds ( $10^{-3}$ seconds) and nanoseconds $\left(10^{-9}\right.$ seconds) to attosecond $\left(10^{-18}\right.$ seconds), zeptoseconds ( $10^{-21}$ seconds), and yoctoseconds $\left(10^{-24}\right.$ seconds) in the third millennium. These increasingly more miniscule measures of time moved from their home of quantum labs and scientific instruments, and seeped into the language of everyday life and corporate-speak.

"We can deliver you high-speed informationqueries by the zeptoseconds!"

"Your package will be with you in 3 to 5 working attoseconds. Thank you for your patience."

"The next capsule will arrive in 1 yoctosecond. Please do not rush to board."

To ask someone to "wait a moment" meant a vastly different request than it is now. As time was portioned into thinner and thinner slices, impatience grew, mistakes became common, and time for reflection shrank. Slowness had always been appreciated, featured in many novels, philosophical manifestos, and movements that saw the liberating power of rest and pause. These counter-movements were, however, drowned out by the gears of capitalism, the lure of convenience, and the possibility of faster and even faster services. The industry that sold Quickness grew (fig. 1).
The logistics industry that ran "24/7" (a depreciated phrase that means "always on") was founded on cheap labourers who were expected to work "mega-shifts" (a continuous shift of more than ten hours) and "ultra-shifts" (a continuous shift of more than a hundred hours).

Likewise, the high-frequency sector swelled in size. It started with "high-frequency traders," stock-brokers that used powerful programs to execute a large number of transactions in fractions of a second. However, "high-frequency" soon became a popular prefix-including "highfrequency delivery drivers," "high-frequency entertainment," "high-frequency administration including but not limited to tax, financial, day care, printing, and filing." In the third millennium, the sands of time were urged to be gushing water, tunnelling through a canal, fast-forwarding through their directed course. Time was linear, forward, and foreclosed.

The more time was divided, the more you can fit in "a moment" you see, and the more it seemed like an extension of life-a complementary service to cryogenic freezing and life extension plans that were trendy at that time. Resources and labour were extracted where possible, to squeeze out any extra fragment of time.

The precision required for this split-second industry, of course, meant that every detail had to be datafied, monitored, and controlledeverything had to be streamlined, lean, and optimised.

\section{EXTRACT FROM CHAPTER 64, "INDUSTRIES"}


WE DELIVER HIGH-SPEED INTERNET

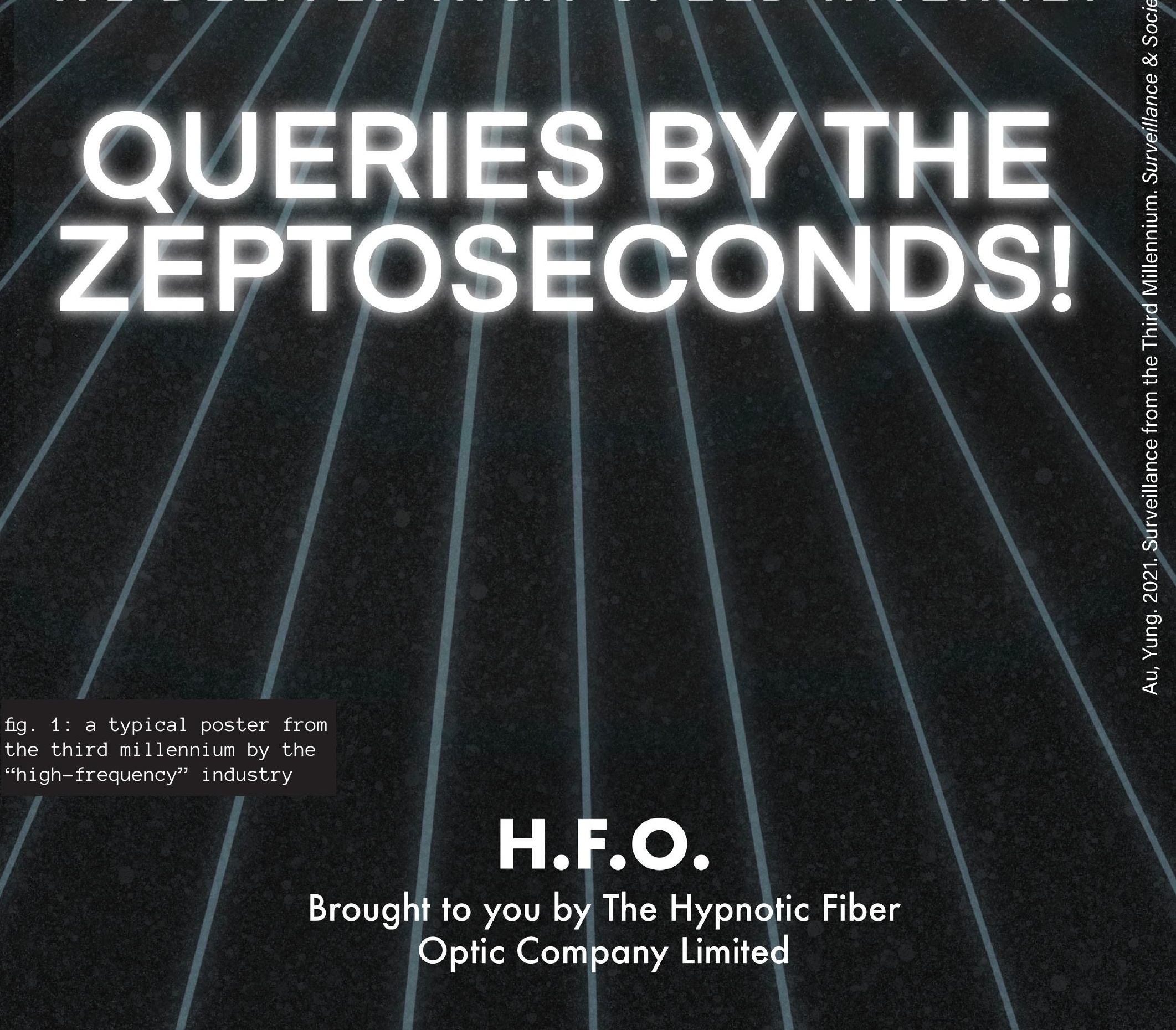




\section{NO MORE}

\section{ANCESTRAL DEBT}

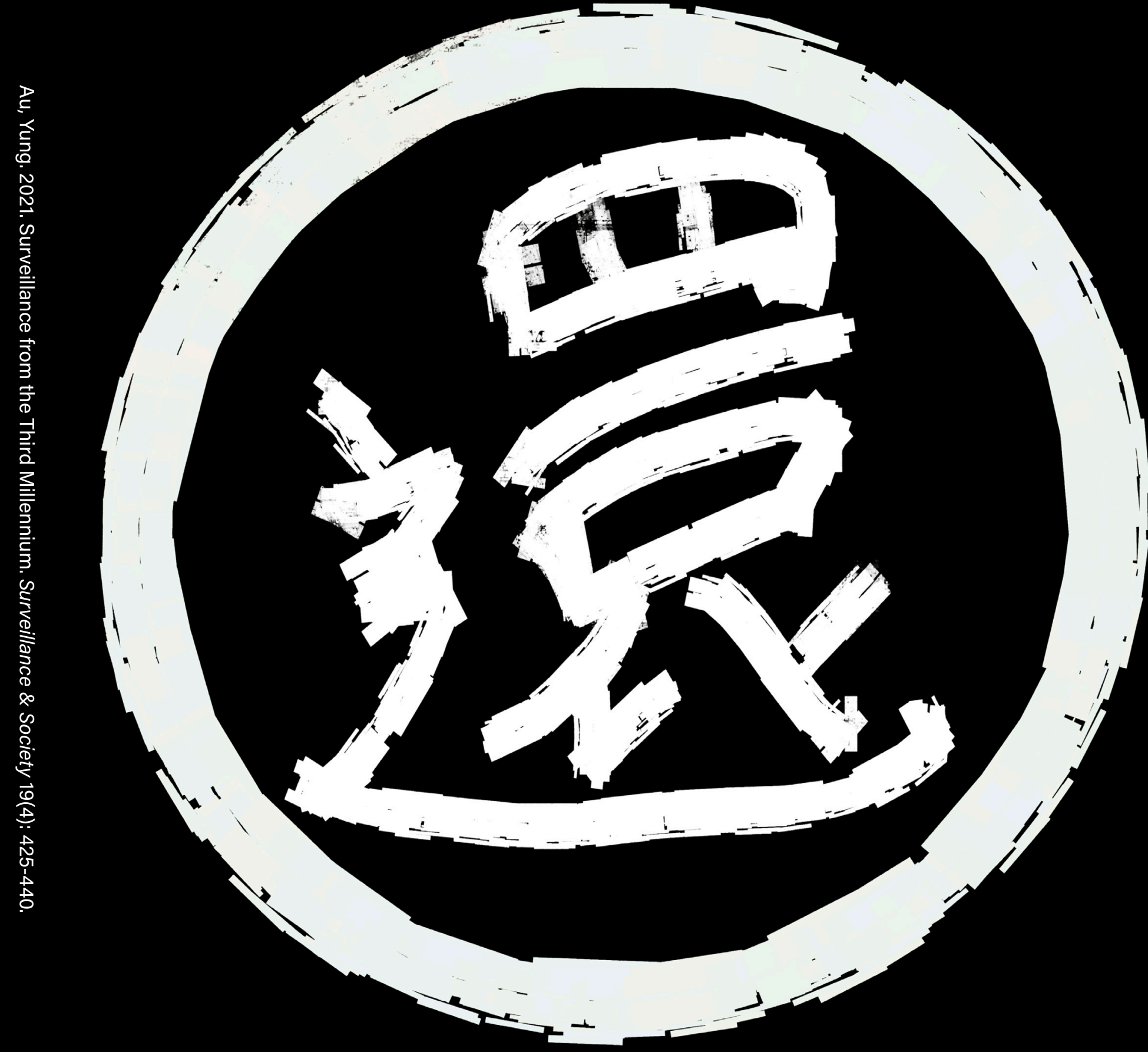

WASH YOUR HANDS OF YOUR PAST, START ANEW 


\section{AN ERA OF PERPETUAL RECORDS}

There was no era in humanoid history that was more surveilled than the third millennium. Anything that could be surveilled, was surveilled. Once the "problem" of data capacities was "solved," the recording of data became exponential. The resulting mountains of information are still being processed to this day. The amount of data reached the limits of language back then. Some sentences that had been used in attempt to surmise the size of accumulated data included:

"A googolplex amount";

"Yoctoseconds of life in all its dimensions, quantified into yottabytes of data";

"We've run out of numbers now";

"A small eternity";

"Hmm...a lot."

Thus, this period is often referred to as the Era of Perpetual Records, the Days of Datafication, or the Surveillant Society.
For example, by mid-millennium, all registered human entities had nano-profiles extending over generations of their family trees, which was useful for tracking any incurred ancestral debt (fig. 2); ${ }^{2}$ for more fine-grained estimations of insurance costs through genetic profiling and families' medical histories; and for predicting life prospects as early as when foetuses were twelve weeks old.

Anything mildly rare or unique (or "deviant") about a person was comprehensively chronicled-anything and everything, from individual smell profiles to brainwave patterns. Even minuscule differences in breathing rhythms, saccadic eye movements, and the split-second biophysical responses to seeing a loved one, was recorded, aggregated, deployed.

While nano-profiling is now seen as backwards, highly invasive, and unnecessarily intimate, those who were not registered back then-and hence, unrecorded-were often worse off. 


\section{OPTIMISE}

\section{Optimise \\ /'optım^ı/}

1. Verb

Make the best or most effective use of (a situation or resource).

e.g. "We manage our time so that we can optimise our productivity."

\section{Computing}

Rearrange or rewrite (data, software, etc.) to improve efficiency of retrieval or processing.

e.g. "The software has been optimised to run 24/7, in perpetuity."

EXTRACT FROM CHAPTER 99921, "TERMINOLOGY" 


\section{Technological Systems \\ When processes are optimized for a certain group of users}

e.g. "Fingerprint recognition systems are optimised for people with fingers."

\section{Legal Term}

Hyper-optimisation. A controversial practice of maximising extraction from people or resources that can often take an inhumane turn.

e.g. "Hyper-optimisation was outlawed in many jursidictions and eventually incorporated into various human rights conventions."

EXTRACT FROM CHAPTER 99921 , "TERMINOLOGY" 


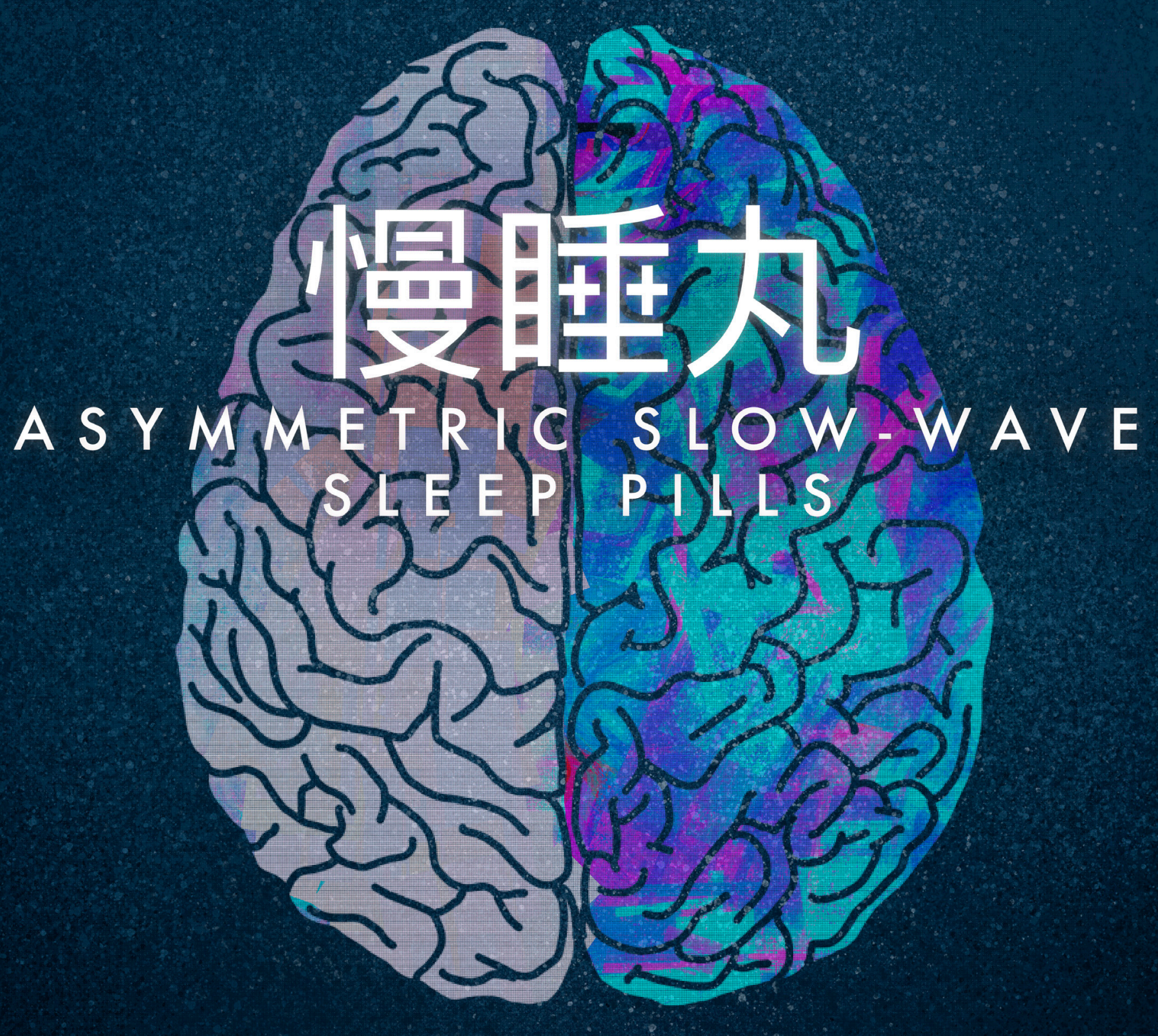

fig. 3: an early advert for Asymmetric Slow-Wave Sleep Pills

\section{YOUR NEW 10,0 O O O Y EAR LIFE BEGINS NOW}

Asymmetric Slow-Wave Sleep Pills (Asym Pills) bring to you all you want, all you need, in tiny, convenient, and cheap capsules. With this pill, you will finally be "awake" for your entire life.

It's simple: this pill lets you be simultaneously awake and asleep through the hemisphere technology inspired by half-brain sleeping animals such as the beluga whale, the peregrine falcon, and the common black bird.
Each pill allows half your brain to be asleep and half your brain to be awake, alert, and productive so that you can reach your full potential. In the Asym World, no one will be left behind - no more "you snooze, you lose." The spirit of Asym is perhaps best captured in Chinese where it is called 慢睡丸 (the slow-sleep pill) or, colloquially, by its homophone, 萬歲丸 (the ten thousand year pill). This is the pinnacle of human longevity. We are proud to welcome you to your new awakening. 


\section{INTRODUCTION TO OUTLAWED TAYLORIST INNOVATIONS}

The outlawing of what were termed Taylorist Innovations only began after mid-millennium-or too late, as some critics observed. At some point, surveillance systems and optimisation systems became one.

Some archetypal Taylorist Innovations include:

(i) Asymmetric Slow-Wave Sleep Pills

(ii) A/B Testing

(iii) Clockmasters

\section{(i) Asymmetric Slow-Wave Sleep Pills}

Asymmetric Slow-Wave Sleep Pills (Asym Pills) were one of the most popular optimising strategies of the third millennium (fig. 3). Asym Pills allowed one hemisphere of a worker's brain to be asleep, while keeping the other hemisphere awake and work-ready. Asym pills, widely considered the pinnacle of Taylorist advances, was inspired by half-brain sleeping animals such as the Amazon River Dolphin, Beluga Whale, Steller Sea Lion, Peregrine Falcon, and the Common Blackbird.

The half-sleep state was mostly induced for what was deemed as "repetitive human labour" such as long-haul driving, call centre services, and factory assembly-line work. These pills made "megashifts" and "ultra-shifts" possible. Corporations came to prescribe these pills as standard practice once they found accident rates to be slightly lower among Asymmed workers (derogatorily called "half-brain workers") when compared to normal, overworked workers. These findings were backed by billions of dollars' worth of experiments conducted in both lab and real work settings-two contexts that began to blur as time went on.

This flawed and fatal logic came crashing down when workplace disasters started snowballing out of control. The specific skillsets required for each of these jobs, honed after years of experience, were finally acknowledged as necessary, and Asym Pills were slowly outlawed towards the end of the millennium. 


\section{(ii) A/B Testing}

Another example of Taylorist Innovations is A/B testing. A/B testing was, most simply, a way to compare two versions of a thing to figure out which performed better.

The method was invented before the third millennium, but its use in mass optimisation was popularised by mega-corporations who wanted to figure out how to influence people at scalewhether it was to get users to buy things or to gamify incentives to extract greater efficiencies from workers. Some popular success metrics from A/B testing included measurements such as "eyeball-time," "information saturation level," and, "degree of work addiction."

At a certain point in the third millennium, workers' rights and users' rights were separated by a thin, superficial line. Everyone was simultaneously a consumer, worker, test subject, and cog in various humming machineries of the 24/7 city. Regardless of whether you were labelled a "customer," "partner," "client," "freelancer," "volunteer," "contributor," "knowledge worker," "sub-contractor," "associate," or "friend": you were, at any given point, the end-user of hundreds of applications and services where you were simultaneously extracted for time, labour, and expertise (whether you knew it or not)-and of course, you were always a data point somewhere. Multiple data points actually.

At some point in the third millennium, it became the norm to be A/B tested throughout your prebirth period, childhood, adult life, work life, and post-mortem life. It became a luxury to be able to opt out of testing (an exclusive service available for a subscription fee of several hundred datacoins per year, an optimised numerical value after generations of $A / B$ testing).

3 For more on Landlords and the Revolt Against Landlords, see p. 937555.

\section{(iii) Clockmasters}

Time continued to be a surveillance tool in the third millennium. Here, Clockmasters had ownership rights over certain stretches of time, much like how Landlords had property rights over certain stretches of land. ${ }^{3}$ During this time, specialised Time Courts became more common for things such as time theft or wage theft, contesting digital timestamps, and challenging various temporal flows.

Nature's cyclic rhythms, whether based on the moon's orbit or by internal biological clocks, were overridden by various corp-times, which were kept by proprietary time instruments, synced calendars, and electronic punch cards. There was a monopoly on time where any "standardised" or "universal" clock-time could be traced back to one of the two data conglomerates at that time.

Time was thus a lucrative investment opportunity (fig. 4). Time and timekeeping was dominated by actors who owned atomic clocks, stop watches, planetary schedules, city alarms, and other seemingly mundane temporal infrastructures.

Time as surveillance also extended to what was called "necropolitics", where some people were classified as sunk costs in terms of optimisation, and it was deemed that there was less and less reason to invest time in them. Life extension plans for getting more bang per hour were reserved for only certain people.

Algorithmic tempos took precedence over natural rhythms. Urban cadences over biological clocks. Time moved differently in the third millennium.

\section{EXTRACT FROM CHAPTER 500, "INNOVATIONS"}




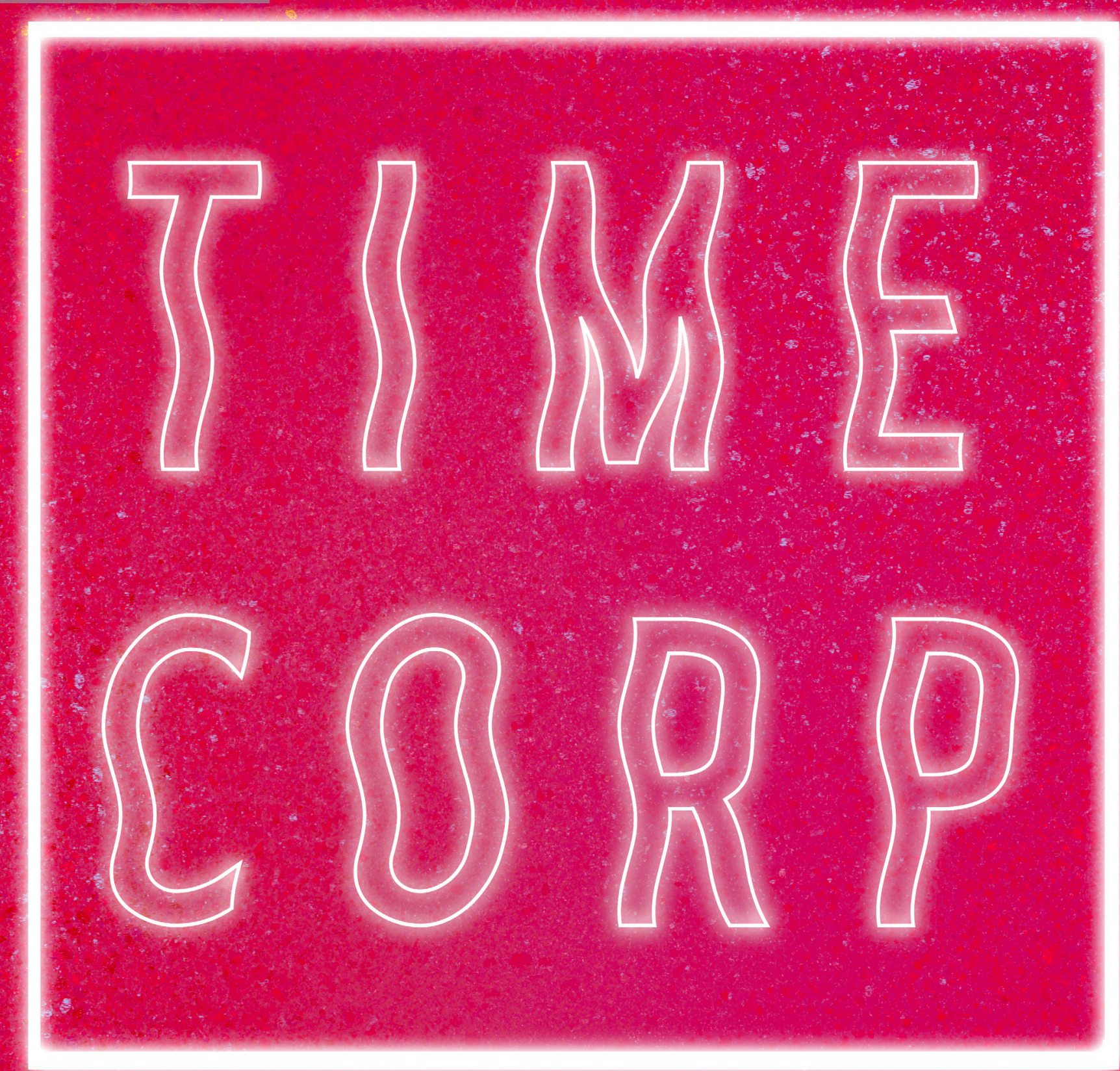

Rewind, remind, review, revise, revisit, revive, relive, return, renew, reset, return, restart....

Time is yours for the taking

Sign up to be a Clockmaster today!

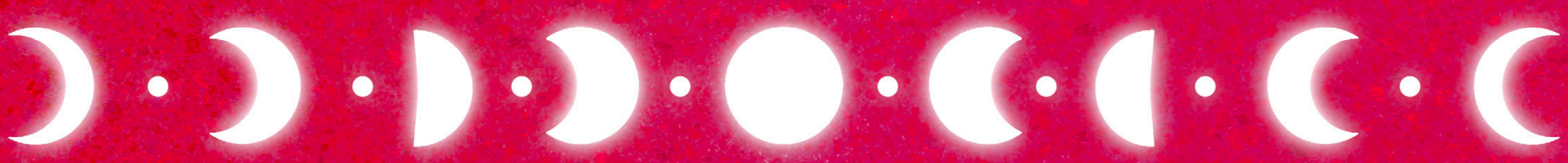




\section{Research Statement}

What will our surveillant futures look like?

This piece prods at this nebulous question by taking an exaggerated look at what would happen if we continued down the pathways to a hyper-datafied society that valued optimisation and quickness above all else.

It puts a fictionalised name and fictionalised systems to ordinary practices that occur today. For instance, the use of time as tools of surveillance and control has been written about extensively, including by E.P. Thompson (1967), who traces the emergence of clock-time as a form of labour discipline in eighteenth-century Europe, to Achille Mbembe (2013), who writes on necropolitics and the power to impose civil or physical death on certain communities, and, more recently, Judy Wajcman (2019), who looks at how Silicon Valley is able to set and accelerate time across the world.

Likewise, this intervention invents fictionalised companies and advertisements on Ancestral Debt, an occurrence that is already happening in some ways, for instance, through Automated DecisionMaking (ADM) systems that ensures certain communities carry the disadvantages of the backgrounds they are born into - whether it is in the processes of prison sentencing algorithms or A-level grade predictions (Benjamin 2019).

It is difficult to assess the moment we are currently living through. It is tempting to describe modern day innovations, problems, and fixations as radical departures from the histories of humankind-that this has never happened before. However, as critical scholars such as Simone Browne (2015; see also, Ogasawara 2019; Semel 2020; Whittaker et al. 2019; Raval 2019) have emphasized time and time again, many of today's problems are an extension of old ones where they run across racial, gendered, class-based, colonial, casteist, ableist, and other lines of marginalisation.

Looking back on our present through a retrospective lens, positioned from a time in an unspecified future, today can be observed as just another period in our long and convoluted histories.

The stretching out of speculative distance can be useful in dispelling ideas of exceptionalism, de-familiarising the familiar, and, perhaps, contributing to an unsettling of a future that is prescribed to us in much of today's discourse.

Acknowledgements: Special and deep thanks to Srujana Katta, Nancy Salem, Lauren Charlton and the special issue team Susan Cahill, Torin Monahan, Bryce Newell and Courtlyn Pippert for their feedback on various parts of this project. 


\section{References}

Benjamin, Ruha. 2019. Race After Technology: Abolitionist Tools for the New Jim Code. Polity.

Browne, Simone. 2015. Dark Matters: On the Surveillance of Blackness. Duke University Press.

Mbembe, Achille. 2013. "Necropolitics." Public Culture 15 (1): 11-40.

Ogasawara, Midori. 2019. "Mainstreaming Colonial Experiences in Surveillance Studies." Surveillance \& Society 17 (5): 726-29.

Raval, Noopur. 2019. "An Agenda for Decolonizing Data Science." Spheres: Journal for Digital Cultures 5: 1-6.

Semel, Beth. 2020. "The Body Audible: From Vocal Biomarkers to a Phrenology of the Throat." Somatosphere (blog). http://somatosphere.net/2020/the-body-audible.html/.

Thompson, E. P. 1967. "Time, Work-Discipline, and Industrial Capitalism." Past \& Present, no. 38: 56-97.

Wajcman, Judy. 2019. "How Silicon Valley Sets Time." New Media \& Society 21 (6): 1272-89. https://doi.org/10.1177/1461444818820073.

Whittaker, Meredith, Meryl Alper, Olin College, Liz Kaziunas, and Meredith Ringel Morris. 2019. "Disability, Bias, and Al." Al Now. 
